\title{
Síndrome del Diente Fisurado: una actualización imprescindible
}

\section{Cracked Tooth Syndrome: an essential update}

Lya del Rosario Magariño Abreus. ${ }^{1}$, Taily Roque Batista. ${ }^{2}$, Lianne Laura de León Ramírez. ${ }^{3} \&$ Ernesto López González. ${ }^{4}$

\begin{abstract}
.
Background: The Cracked Tooth Syndrome has increased its incidence worldwide and constitutes a fundamental diagnostic challenge in dental clinical practice. In Cuba it is not included in the current classification of dental trauma, so it is not diagnosed or treated. Objective: to describe the Cracked Tooth Syndrome, as a frequent stomatological entity. Methodology: A bibliographic review was carried out taking into account the scientific literature in Spanish and

\section{Resumen.}

Introducción: El Síndrome del Diente Fisurado ha aumentado su incidencia a nivel mundial y constituye un desafío diagnóstico fundamental en la práctica clínica estomatológica. En Cuba no está incluido en la clasificación vigente de traumatología dental por lo que no se diagnostica ni se trata. Objetivo: describir el Síndrome del Diente Fisurado, como entidad estomatológica frecuente. Metodología: Se realizó una revisión bibliográfica teniendo en cuenta

\footnotetext{
${ }^{1}$ Universidad de Ciencias Médicas de Cienfuegos. Cienfuegos, Cuba, lya.magarino2000@gmail.com https://orcid.org/0000-0002-9933-5323

2 Universidad de Ciencias Médicas de Cienfuegos. Cienfuegos, Cuba. tailyroque@gmail.com https://orcid.org/0000-0003-0274-6028

3 Universidad de Ciencias Médicas de Matanzas. Matanzas, Cuba, , liannelaura@nauta.cu https://orcid.org/0000-0001-9250-1889

${ }^{4}$ Universidad de Ciencias Médicas de Cienfuegos, Departamento de Investigaciones. Cienfuegos, Cuba. asesorf@ucm.cfg.sld.cu https://orcid.org/0000-0001-6607-7658
} 
English, including all original research articles, review articles and case reports, using the Scielo, PubMed, and Scopus databases. Around 63 articles were identified, of which 54 were selected according to the academic criteria of the Cuban Stomatological School, of which $50 \%$ were updated. The information was analyzed and integrated. Results: Topographically, the teeth of the posterior sector were the most affected. It mainly affects adults in the age range of 30 to 60 years; while sex is not a determining variable in its incidence. Its essential cause is the incomplete vertical fracture or fissure in the dentin, which are not usually shown on the X-ray. Conclusions: It was concluded that despite the fact that this syndrome is a common and welldocumented clinical entity in several countries as one of the traumatic conditions that can evolve into pulp necrosis, in Cuba, there is no protocol that regulates its identification.

Keywords: Cracked Tooth Syndrome, dental fissure, green stem fracture, incomplete vertical fracture. la literatura científica en idioma español e inglés, incluyéndose todos los artículos de investigación originales, artículos de revisión e informes de casos, utilizando las bases de datos Scielo, PubMed, Scopus. Se identificaron alrededor de 63 artículos, de los cuales fueron seleccionados 54 según los criterios académicos de la Escuela Estomatológica Cubana, de ellos el 50\% actualizado. Se analizó e integró la información. Resultados: Topográficamente, los dientes del sector posterior resultaron ser los más afectados. Afecta fundamentalmente a adultos en el rango de edad de 30 a 60 años; mientras que el sexo no es una variable determinante en su incidencia. Su causa esencial es la fractura vertical incompleta o fisura en la dentina, que no suelen ser mostradas en la radiografía. Conclusiones: Se concluyó que a pesar de que este síndrome es una entidad clínica común y bien documentada en varios países como una de las afecciones traumáticas que puede evolucionar a una necrosis pulpar, en Cuba, no existe ningún protocolo que regule su identificación.

Palabras claves: Síndrome del Diente Fisurado, fisura dentaria, fractura en tallo verde, fractura vertical incompleta.

\section{Introducción.}

El Síndrome del Diente Fisurado o Agrietado (SDF), clasificado dentro de la traumatología dental, es una de las primeras causas de extracción dentaria debido a las dificultades para su detección, y quizás la patología bucal peor diagnosticada. De acuerdo a la Asociación Dental Americana (ADA, 2018), en su clasificación más actual, lo define como una colección de síntomas caracterizados por dolor agudo transitorio experimentado al masticar, y designa a la fractura incompleta dentaria o fisura, el signo que origina la entidad. En el 2017, en la Revista Dental Británica describió al diente 
fisurado como un plano de fractura de profundidad desconocida que se origina desde la corona, pasa a través de la estructura dentaria, se extiende subgingivalmente y puede progresar hasta conectar el espacio pulpar y/o el ligamento periodontal. (Banerji, 2017, p.10).

Álvarez (2015) plantea: "se utilizan otros términos para su denominación como: fractura incompleta corono-radicular, línea de fractura, fractura fisural, hendidura y fractura en tallo verde". p. 1

No es un concepto nuevo, se estudia ampliamente desde la década de los 50 cuando Gibbs describió por primera vez los síntomas clínicos de fractura incompleta de dientes posteriores y la denominó "odontalgia de fractura cuspídea". Sin embargo, no fue hasta 1964, que el SDF fue acuñado y descrito por Cameron, basándose en que la condición dental presentaba un conjunto de signos y síntomas complejos que se manifestaban simultáneamente (Cameron, 1964) (Herrera, 2015).

De acuerdo a la Organización Mundial de la Salud (como se citó en Petti, Glendor y Andersson, 2018), las fisuras que causan el SDF se clasifican como fracturas coronarias dentro de los traumatismos dentales; estos últimos se consideran una de las siete causas de morbilidad por enfermedades y trastornos bucodentales, y su prevalencia en las denticiones temporal y permanentes aproximadamente el $20 \%$.

Datos epidemiológicos demuestran que las fisuras son el tercer motivo más frecuente de pérdida de piezas dentales, alcanzando el $25 \%$ en los países industrializados, y una de las tres condiciones dentarias fundamentales que causan dolor (Banerji, 2017) (Geurtsen, Gunay y Schwarze, 2006). Son limitados los reportes de prevalencia en niños y adolescentes, mientras que los valores en los adultos se encuentran entre un $34 \%$ y un 74 $\%$ con un gran aumento en los últimos años relacionado con los altos niveles de estrés de la población (Weisburd, Giménez, Gutiérrez, Tomaghelli y Perdomo, 2017) (Chalas y Hanni, 2019).

En Cuba los estudios de incidencia son contradictorios. Bajo la Resolución Ministerial No. 226, se norma la práctica de las afecciones endodónticas, protocolizadas a través de las Guías Prácticas Estomatológicas, constituyendo una normativa general que cubre todo el territorio nacional. Está vigente la clasificación antigua de lesiones traumáticas del noruego Ingeborg Jacobsen por ser más sencilla y didáctica, publicadas desde 2003, que, además, están basadas en las Normas de Endodoncia del año 1983 (González, Garmendia, Granados y Martínez, 2003). Resulta que no permite clasificar, y por tanto diagnosticar, un conjunto de entidades erróneamente consideradas como signos aislados de patologías sí reconocidas por nuestra comunidad científica, como es el caso del SDF. Esta situación conlleva inevitablemente a errores diagnósticos y terapéuticos. En investigaciones nacionales se ha podido determinar un número elevado de tratamientos pulpares radicales que han podido ser evitados si hubieran sido diagnosticados y tratados como alguna de las entidades que conforman al complejo síndrome. 
A nivel internacional, en los últimos años la producción intelectual al respecto ha sido copiosa, sin embargo, el término del "síndrome del diente fisurado" aún es engañoso ya que hay una serie de síntomas que no forman un patrón distinto y confiable, pues estos variarán con los dientes que tienen pulpas sanas, los que tienen pulpa necrótica o inflamada y los dientes que han sido tratados desde la raíz (Khaler, 2008).

Es por ello que el objetivo de este trabajo es describir el Síndrome del Diente Fisurado, como entidad estomatológica frecuente, pues los odontólogos deben conocer las características clínicas de esta entidad, los avances en los métodos diagnósticos y su etiología, para poder ofrecer al paciente el tratamiento adecuado.

\section{Metodologia.}

Se realizó una revisión bibliográfica teniendo en cuenta la literatura científica en idioma español e inglés, incluyéndose todos los artículos de investigación originales, artículos de revisión e informes de casos, utilizando las bases de datos Scielo, PubMed, Scopus. Se identificaron alrededor de 63 artículos, de los cuales fueron seleccionados 54 según los criterios académicos de la Escuela Estomatológica Cubana, de ellos el 50\% actualizado. Se analizó e integró la información.

\section{Resultados.}

De acuerdo con la Asociación Internacional del Estudio del Dolor (como se citó en Gutmann y Lovdahl, 2011), el SDF es un dolor breve y agudo en un diente, frecuentemente no diagnosticado hasta que una parte del diente se fractura o la entidad se hace evidente. Mientras que una revisión publicada por la Revista de la Sociedad Española del Dolor, plantea que la dificultad en el manejo de este dolor estriba en que la detección de la fractura y su profundidad son difíciles de valorar, ya que no existe una pérdida estructural o separación visible de la estructura dentaria (Migueláñez, Goicochea, López y Martínez, 2019).

Dientes afectados frecuentemente:

Topográficamente, en todos los estudios consultados, los dientes del sector posterior, es decir, molares y premolares, resultaron ser los más afectados, difiriendo solamente en su localización en la arcada. Se acepta que el diente más incidente es el primer molar inferior y los menos incidentes los premolares inferiores (Álvarez, Clavera, Martínez, 2015) (Herrera, 2015) (Moradas, 2016) (Sin-Young, Su-Hyun, Soo-Bin, Gyung-Ok y SungEun, 2013) Solo difieren dos investigaciones, pues señalan al segundo molar inferior como el diente más afectado (Kang, Kim y Kim, 2016) (Sung-Eun, A-Ra y Sin-Young, 2017).

Al primer molar inferior se le atribuye una alta incidencia debido a la prominencia de la cúspide mesio-palatina del primer molar superior, la cual ejerce un efecto de constante debilitamiento sobre él (Álvarez, Clavera, Becerra y Rodríguez, 2014). Delgado (2013) 
afianza esta teoría alegando que al ser los primeros dientes en erupcionar, son los primeros en los que se realiza operatoria dental con restauraciones amplias llegando a debilitarlos.

Se asume que es más frecuente encontrarse con fracturas incompletas en dientes cariados o muy restaurados (incluso con restauraciones conservativas), pero también pueden afectarse los dientes sanos (Lynch, y McConell, 2002) (Mallqui y Hernández, 2012) (SinYoung, Su-Hyun, Soo-Bin, Gyung-Ok y Sung-Eun, 2013) (Rosen, 2012) (González y López, 2018) (Sánchez, Jiménez y Gale, 2018) (Krell y Caplan, 2018).

Cerca del $82 \%$ de SDF ocurre en dientes restaurados con amalgama. Es interesante destacar que puede afectar múltiples dientes en el mismo paciente (Batalha, Gondo y Stolf, 2014).

Edad: En general la bibliografía consultada señala que afecta fundamentalmente a adultos en el rango de edad de 30 a 60 años (Kang, Kim y Kim, 2016) (Sung-Eun, A-Ra y Sin-Young, 2017)

Sin embargo, Fitzpatriek observó que fracturas incompletas ocurrieron principalmente en personas entre 30-39 años; del mismo modo, fue reportado un caso de SDF unilateral en una paciente femenina de 26 años; estos hallazgos indican que cada vez más pacientes jóvenes son afectados (Martins, Faquim, Batista, 2019).

Sexo: Actualmente, los autores concuerdan en que el sexo no es una variable determinante en la incidencia del SDF (Herrera, 2015) (Moradas, 2016) (Álvarez et al., 2014).

Raza: En pacientes de raza asiática se han reportado casos con presencia del Síndrome en los primeros molares mandibulares aparentemente sanos; sin embargo, la razón de predilección racial es todavía desconocida; se han sugerido la dieta, los hábitos de apretamiento y otros hábitos dañinos como morder huesos durante la masticación de alimentos (Chan, Lin, Tseng, y Huei, 2018).

Etiología: En cuanto a los factores causales del SDF existen diversos criterios. La mayoría de los autores coincide en que la causa esencial es la fractura vertical incompleta o fisura en la dentina, la cual se dirige hacia la raíz dentaria (Álvarez et al., 2015) (Herrera, 2015).

Otros aseguran que la etiología es multifactorial. Las fisuras, por su parte, son el resultado de fuerzas que exceden el límite elástico de la dentina y por tanto ocasionan el resquebrajamiento de la misma (Weisburd et al., 2017).

Lynch (2002) dividió las causas en cuatro grupos principales:

1. Procedimientos restauradores: pines de fricción, colocación de composite sin una técnica depurada de capas, restauración protésica con coronas y puentes en las que se ha realizado un tallado excesivo, restauraciones de mala calidad, 
2. Factores oclusales: accidente masticatorio con algún objeto duro, el trauma oclusal, las interferencias oclusales, los hábitos parafuncionales o el bruxismo, principalmente nocturno y excéntrico. Así, las fisuras dentarias se encuentran en pacientes que muerden vigorosa o repentinamente alimentos u objetos duros, tales como hielo, semillas, dulces duros, lápices, uñas, clavos, etcétera. Numerosos autores coinciden con estos factores, considerándolos, en ocasiones los más importantes (Gonzáles y López, 2018) (Feng, 2017).

Hay quien encuentra relación con ciertas profesiones que predisponen a momentos de estrés dental, apretamiento o malos hábitos: jugadores de fútbol, boxeadores, músicos de instrumentos de viento, etc.

3. Factores anatómicos y de desarrollo: áreas de debilidad por displasia cualitativa de los tejidos dentarios, morfología abrupta y marcada por surcos, fosas y fisuras; e inclinación y altura de las cúspides.

4. Otros factores: la edad constituye un factor predisponente, pues se produce un paulatino envejecimiento dentario y de los tejidos por la pérdida de elasticidad de los mismos.

Se han reportado varios casos de fisuras dentarias en pacientes que usan los body piercing en la lengua, labios y carrillos. No pocos autores afianzan esta teoría, aconsejando no usar piercing dental u otros objetos en la boca, por ser posibles factores precipitantes (Moradas, 2016) (Deog-Gyu, Yi, Su-Jung y Jeong-Won, 2015).

Cambios térmicos: Cuando un diente es repentinamente expuesto a bajas temperaturas el esmalte trata de contraerse, esto puede traer como resultado la aparición de fisuras en el esmalte o la extensión de las ya existentes.

Diagnóstico: El diagnóstico diferencial incluye grietas en el esmalte solamente, grietas verticales y fracturas incompletas. Es una patología común con síntomas y signos característicos, pero en ocasiones, coincidentes con otras afecciones dentales como caries profunda, endodoncia fracasada, presencia de conductos accesorios, periodontitis periapical y sinusitis temporomandibular (Sebeena, Boopathi y Arjun, 2012).

Se conoce que las fisuras dentarias pueden permanecer asintomáticas u originar una sintomatología muy variada; la cual se mantiene presente por períodos que oscilan entre semanas y meses. La totalidad de los artículos consultados refiere que los síntomas tempranos son dolor súbito, que es agudo al masticar o al morder y puede aparecer al ocluir, en movimientos excursivos y en algunos casos al rebote; además de sensibilidad térmica principalmente al frío; no se observan alteraciones radiográficas y hay dificultad para identificar el diente causante (Yaping, Gongpei y Fang, 2019).

Esta sintomatología puede variar de acuerdo al grado de compromiso pulpar, de ahí que la ausencia de síntomas en determinados pacientes, pueda ser explicada debido a una lenta degeneración de la pulpa, produciéndose necrosis. Del mismo modo, West (2008) lo describió como una condición de dolor que se puede confundir con la hipersensibilidad 
de la dentina porque a veces es extremadamente difícil ver el crack inicial. Un factor determinante para el diagnóstico diferencial es que el dolor del síndrome tiende a ser más la liberación de presión que el aumento de la presión oclusal.

La Historia Clínica es considerada por algunos investigadores como el paso inicial el más importante para guiar el diagnóstico, refiriendo que el comentario más determinante es: "el diente me duele cuando muerdo". Lo siguiente sería realizar el examen clínico visual (Filipi, 2018).

Posteriormente se realizan otros métodos que varían según la impresión diagnóstica y el centro estomatológico. Por ejemplo, la transiluminación con la ayuda de un aparato de fibra óptica, de tal forma que se iluminará el fragmento del diente hasta la línea de fractura, si la hubiera (Migueláñez, Goicochea, López y Martínez, 2019) (González y López, 2018).

También es muy popular el uso de colorantes, como el azul de metileno o violeta cristal para destacar las líneas de fractura, pero se ha visto que requieren varias aplicaciones para ser efectivos y necesitan restauraciones provisionales. Investigadores añaden el empleo de yodo que hace evidente la fisura en forma de una línea oscura (Mallqui y Hernández, 2012) (Migueláñez et al., 2019) (Filipi, 2018).

Desafortunadamente estos métodos no brindan información certera acerca de la profundidad de la misma. Sin embargo, numerosos profesionales apuestan por su uso complementario como Ojeda Suárez (2016), utilizando cloruro de metiltionina y microscopio (Ojeda, Llanas y Rodríguez, 2016).

El Test de mordida cuenta con gran aceptación en el gremio estomatológico, pues simula la oclusión y así reconoce los síntomas asociados con fracturas incompletas; no obstante, no siempre es útil, ya que pierde fiabilidad si no se concentran las fuerzas en una cúspide y puede causar, a la vez, una fractura cuspídea (Moradas, 2016).

Similar a esta forma de detección, se dispone de varios elementos como: rollos de algodón, discos de goma o el eyector de saliva, para reproducir la molestia a la masticación. Lo más avanzado en este sentido es el dispositivo "Tooth Slooth", que resultó ser una herramienta útil y precisa para reproducir el dolor de mordedura, contribuyendo al diagnóstico temprano del diente fisurado (Yaping, Gongpei y Fang, 2019).

Son aplicados igualmente los test de vitalidad, que en los dientes afectados suelen ser positivos, aunque con el tiempo puede ser negativo, debido a una progresiva degeneración de la pulpa (Hilton, 2018). En Cuba es empleado el vitalómetro o pulpavitalómetro. En el caso de compromiso periodontal el sondaje homónimo puede revelar la gravedad de la fractura. 
Cuando el estado del diente es aún más grave se emplea la cirugía exploratoria para evidenciar, sobre todo, fracturas radiculares verticales acompañadas de pérdida ósea (Delgado y Afrashtehfar, 2013).

Quizás la mayor desventaja para el diagnóstico es que la radiografía no suele mostrar las fracturas dentarias incompletas; por lo que tendrán un uso limitado cuando el Síndrome presenta fracturas en dirección mesiodistal paralelas al plano de la película -las más frecuentes-; sin embargo, serán útiles en fracturas más raras, en dirección bucolingual. Solo cuando el haz de rayos $\mathrm{X}$ pasa directamente a través de la línea de fisura es cuando se podrán identificar este tipo de fracturas; es por ello que se recomiendan varias angulaciones en la película cuando se trate de identificar una fractura vertical (Delbem, Cunha, Percinoto y Da Silva, 2018).

Recientemente se emplea el Cone Beam Computed Tomography (CBTC), como ayuda de la radiología para el diagnóstico, no obstante, incluso con esta tecnología, muchas veces es muy difícil lograr la detección de una fisura radicular. Un estudio de 2019 refuerza su relevancia, declarando su uso para detectar fracturas longitudinales, y añade el empleo de técnicas recientes que utilizan tomografía de coherencia óptima (OCT), por sus siglas en inglés, para detectar líneas de fisura invisibles al ojo humano (Martins et al., 2019).

Estas nuevas técnicas se emplean en conjunto por varios estomatólogos para mejorar la calidad del diagnóstico.

Tratamiento: Se conoce que la mayoría de las decisiones clínicas tomadas para el tratamiento no se basan en evidencias científicas, a pesar de que se sugieren varias alternativas. En cuanto a los métodos terapéuticos es mundialmente conocido que la vía más rápida, aunque no más sencilla ni conservadora, para cesar la sintomatología del paciente es el tratamiento del sistema de conductos radiculares Excepto en el caso de enfermedad pulpar/apical, que estos dientes requieren tratamiento no quirúrgico del conducto radicular (Alkhalifah, Alkandari, Sharma y Moule, 2017) (Dow, 2016).

Durante la fase de obturación del sistema de conductos radiculares, en todos los artículos consultados se recomienda emplear una mínima fuerza de condensación y la obturación con gutapercha termoreblandecida o ionómero de vidrio. Como medicación intraconductos se aconseja la colocación de hidróxido de calcio ya que medicamentos como el eugenol o el formocresol pueden pasar a través de la línea de fisura y afectar adversamente el periodonto. Asimismo, se emplean, para la inmovilización inmediata extracoronalmente, bandas de ortodoncia bien ajustadas y cementadas sin interferir en la oclusión, aunque persiste la sensibilidad térmica. Estudios sugieren la colocación de una protección provisional acrílica en lugar de la banda ortodóncica. También se realizan ajustes oclusales en los dientes afectados para reducir las fuerzas ejercidas sobre él, previniendo así la propagación de la fractura (Herrera, 2015) (León, Arada, López y Armas, 2012). 
En el apartado de los procedimientos restauradores existen múltiples materiales que son seleccionados por el estomatólogo de acuerdo al daño dental. Se ha descrito la preparación de coronas acrílicas provisionales como una opción para el tratamiento inmediato de fracturas incompletas, pero, debido a su complejidad no se recomienda su uso, más cuando, una vez confeccionadas, la sintomatología puede que no cese (Moradas, 2016).

Por otro lado, un tratamiento inmediato y novedoso es la colocación de resina compuesta, llamada ferulización directa con composite, que se une directamente sobre la superficie del diente a ferulizar a través de la fractura e inmoviliza los segmentos. Se ha descrito como las restauraciones con composite pueden alcanzar una resistencia a la fractura mayor incluso que los dientes sanos (Martins et al., 2019).

Por otra parte, las amalgamas convencionales no tienen un potencial adhesivo intrínseco por lo que tienen poca capacidad de interferir sobre las fracturas incompletas. No obstante, se describe la restauración con onlays de amalgama (restauraciones con protección cuspídea o corona completa), principalmente en tratamientos primarios de los dientes afectados, pueden ser más agresivas en cuanto al desgaste, pero resultan mejor adaptadas y más pasivas.

Actualmente es electiva una técnica para tratar dientes con esta afección utilizando resinas fluidas. Este tipo de material tiene la ventaja que puede ser inyectado dentro de la línea de fisura, previo grabado ácido, y crea una traba mecánica que impide la profundización de la fisura (Soares de Toubes, Moreira y Cota, 2020).

El estudio más reciente, publicado por la Revista Iraní de Endodoncia asevera que la literatura es escasa en protocolos de cómo resolver SDF usando restauraciones cerámicas hechas por el sistema (CAD-CAM), por sus siglas en inglés: diseño asistido por computadora y fabricación asistida por computadora; el cual proporciona un tratamiento restaurador rápido y eficiente, generalmente en una sola visita, lo que reduce el riesgo de contaminación y microfiltración de la línea agrietada. Cuando hay comprometimiento pulpar se realiza el tratamiento endodóntico fisura (Soares de Toubes et al., 2020).

Desafortunadamente, en la mayoría de los casos el tratamiento suele ser la extracción del diente en cuestión, debido al desconocimiento de esta patología por parte de los profesionales, al no tener claro el proceso evolutivo, ni el establecer las medidas terapéuticas adecuadas.

Una terapia opcional es el control de hábitos parafuncionales: El grado de stress emocional tiene una influencia determinante en el bruxismo, por tano el tratamiento debe ser integral, tanto la colocación de una férula oclusal como la medicación son importantes. 


\section{Conclusiones.}

- El SDF es una entidad clínica común y bien documentada en varios países, en Cuba ni siquiera se encuentra dentro de la clasificación de traumatología dental vigente, por lo tanto, no se diagnostica ni se trata.

- Constituye la patología bucal peor diagnosticada debido a la dificultad para identificar el crack inicial y la semiología propia, debido a ello han surgido varios avances tecnológicos para suplir esta dificultad.

- Se recomienda el estudio del tema y la realización de acciones por los organismos pertinentes para incluirla en la clasificación actual, pues es una de las afecciones traumáticas más frecuentes que puede acarrear resultados negativos como la necrosis pulpar.

\section{Referencias bibliográficas.}

Alkhalifah, S., Alkandari, H., Sharma, P.N., y Moule, A.J. (2017)Treatment of Cracked Teeth. $J$ Endod, 43(9), 1579-1586. Recuperado de https://pubmed.ncbi.nlm.nih.gov/28734650/

Álvarez, J., Clavera, T.J., y Martínez, D. (2015). Actualización de aspectos relacionados con el Síndrome del Diente Fisurado. Rev haban cienc méd,14(4), 397-408. Recuperado de http://scielo.sld.cu/scielo.php?script=sci_arttext\&pid=S1729519X2015000400004\&Ing=es. 14(4):397-408.

American Dental Association. (2018). Cracked Tooth Syndrome. Recuperado de https://www.ada.org/en/publications/cdt/glossary-of-dental-clinical-andadministrative-ter

Avendaño, A. (2017). El Síndrome del Diente Fisurado: Etiología, Diagnóstico y Tratamiento (tesis de pregrado). Universidad Central de Venezuela, Venezuela.

Banerji, S. Metha S.B., y Millar B.J. (2017). The management of cracked tooth syndrome in dental practice. $\mathrm{Br}$ Dent $J$, 222(9), 659-666. Recuperado de https://doi.org/10.1038/sj.bdj.2017.398

Batalha, S., Gondo, R., y Stolf, S. C. (2014). Cracked Tooth Syndrome in an Unrestored Maxillary Premolar: A Case Report. Operative Dentistry, 39(5), 460-468. Recuperado de https://pubmed.ncbi.nlm.nih.gov/24517730/

Cameron, C.E. (1964). Cracked tooth syndrome. J Am Dent Assoc, 68(3), 405-411. Recuperado de https://pubmed.ncbi.nlm.gov/61220/

Chalas, R., y Hanni, S. (2019). Cracked Tooth Syndrome, management of dental emergencies in children and adolescents. Estados Unidos: Wiley Online Library. Recuperado de https://onlinelibrary.wiley.com/doi/abs/10.1002/9781119372684.ch7.3 
Chan, Ch., Lin, Ch., Tseng, S., y Huei, J. (2018). Vertical root fracture in endodontically versus nonendodontically treated teeth. A survey of 315 cases in Chinese patients. Oral Surg Oral Med Oral Pathol, 87, 504-7. Recuperado de https://www.oooojournal.net/article/S1079-2104(99)70252-0/pdf

D'angelis, A. (2007). The lingual barbell: a new etiology for the cracked tooth syndrome. The Journal of American Dental Association, 128, 1438-9. Recuperado de https://pubmed.ncbi.nlm.nih.gov/9332146/

Delbem, A., Cunha, R., Percinoto, C., y Da Silva, L. (2018). Severe lateral luxation and root fracture; report of a case with 5-year follow-up. Endodontics \& Dental Traumatology, 15, 91-93. Recuperado de https://onlinelibrary.wiley.com/doi/pdf/10.1111/j.1600-9657.1999.tb00761.x

Delgado, M., y Afrashtehfar, K. (2013). Concepto actual y caso clínico. Odontología actual, 10(124), 42-44. Recuperado de https://www.researchgate.net/publication/260787229_Diente_fisurado_Cocepto _actual_y_caso_clinico

Deog-Gyu, S., Yi, Y., Su-Jung, S., y Jeong-Won, P. (2015). Analysis of Factors Associated with Cracked Teeth. J Endod, 38, 288-292. Recuperado de https://www.jendodon.com/article/S0099-2399(11)01379-3/abstract

Dow, D.M. (2016). Outcome of endodontically treated cracked teeth (tesis doctoral). Faculty of the Endodontics: University of the Health Sciences, Estados Unidos.

Feng, Q. (2017). Cracked Teeth and Poor Oral Masticatory Habits: A Matched Casecontrol Study in China. Journal of Endodontics, 6(43), 885-889. Recuperado de https://www.sciencedirect.com/science/article/pii/S0099239917300122

Filipi, A. (2018). Transplantation of displaced and dilacerated anterior teeth. Endodontics \& Dental Traumatology, 14, 93-98. Recuperado de https://pubmed.ncbi.nlm.nih.gov/9558523/

Geurtsen, W., Gunay, H., y Schwarze, T. (2006) Diagnóstico, tratamiento y prevención del Síndrome del Diente Fisurado. Dialnet, 4(19), 189-197. Recuperado de https://dialnet.uniroja.es/servlet/articulo?codigo $=1957662$

González, C.D., y López, A.Y. (2018) Efectividad de los compuestos para el tratamiento de la sensibilidad dental: una revisión sistemática (tesis de maestría). Facultad de Odontología: Universidad Santo Tomás, Bucaramanga.

González, G., Garmendia, G., Granados, A., y Beauballet, B. (2003). Guías prácticas clínicas ante traumatismos dentarios y faciales. En Colectivo de autores (Ed.), Guías prácticas de Estomatología (pp. 128-194). La Habana, Cuba: Editorial Ciencias Médicas. 
Gutmann, J.L., y Lovdahl, P. (2011). Problem solving in Endodontics. Recuperado de https://www.elsevier.com/books/problem-solving-inendodontics/9780323068888

Herrera, J. V. (2015). Manejo del Dolor Aplicado a la Odontología (tesis de maestría). Facultad Piloto de Odontología: Universidad de Guayaquil, Ecuador.

Hilton, T.J. (2018). Associations of types of pain with crack level, tooth-level and patientlevel characteristics in posterior teeth with visible cracks: Findings from the National Dental Practice - Based Research Network. Journal of Dentistry, 70(1), 67-73. Recuperado de https://pubmed.ncbi.nlm.nih.gov/29289728/

Kang, S.H., Kim, B.S., y Kim, Y. (2016). Cracked teeth distribution, characteristics, and survival after root canal treatment. J Endod, 42(4), 557-62. Recuperado de https://www.sciencedirect.com/science/article/pii/S0099239916000662

Khaler, W. (2008). The cracked tooth conundrum: Terminology, classification, diagnosis, and management. Am J Dent, 21, 275-282. Recuperado de https://www.amjdent.com/Archive/ReviewArticles/2008/Khaler\%20AJD\%2010 -08.pdf

Krell, K.V., y Caplan, D.J. (2018). 12-month Success of Cracked Teeth Treated with Orthograde Root Canal Treatment. J Endod, 44(4), 543-548. Recuperado de https://pubmed.ncbi.nlm.nih.gov/29429822/

León, M., Arada, J.A., López, M.N., y Armas, D. (2012). Traumatismos dentarios en el menor de 19 años. Rev Ciencias Médicas de Pinar del Río, 16(5), 4-13. Recuperado de http://publicaciones.pri.sld.cu/rev-fcm/rev-fcm165/010512.htm

Lynch, C. D., y McConell, R. J. (2002). The Cracked Tooth Syndrome. J Can Dent Assoc, 68(8), 470-5. Recuperado de https://www.cda-adc.ca/jcda/vol-68/issue-8/470.pdf

Mallqui, L.L., y Hernández, J.F. (2012). Traumatismos dentales en dentición permanente. Revista Estomatológica Herediana, 1(22), 32-35. Recuperado de http://www.redalyc.org/articulo.oa?id=421539367008

Martins, M., Faquim, P., y Batista, J. (2019). Diagnóstico, plan de tratamiento y restauración de un diente con el síndrome del diente fisurado: reporte de caso y seguimiento de siete años. Rev Estomatol Herediana, 29(2), 33-34. Recuperado de https://www.scielo.org.pe/scielo.php?script=sci_arttext\&pid=S101943552019000200007\&Ing=es.

Migueláñez, B., Goicochea, C., López, A., y Martínez, M.A. (2019). Dolor orofacial en la clínica odontológica. Rev Soc Esp Dolor, 26(4), 233-242. Recuperado de http://scielo.isciii.es/pdf/dolor/v26n4/1134-8046-dolor-26-04-00233.pdf

Moradas, M. (2016). Síndrome del diente agrietado-fisurado. Revisión bibliográfica para la toma de decisiones. MX Maxillaris. Recuperado de 
https:/www.maxillaris.com/foro-20161102-Sindrome-del-diente-agrietadofisurado-Revision-bibliografica-para-la-toma-de-decisiones.aspx

Ojeda, J.F., Llanas, J.U., y Rodríguez, I. (2016). Tratamiento endodóntico en un diente con el Síndrome del Diente Fisurado. Reporte de un caso. Revista Mexicana de Estomatología, 2(3), 188-189. Recuperado de https://www.remexesto.com/index.php/remexesto/article/view102

Petti, S., Glendor, U., y Andersson, L. (2018). World traumatic dental injury prevalence and incidence, a meta-analysis- One billion living people have had traumatic dental injuries. Dent Traumatol, 34(2), 71-86. Recuperado de https://pubmed.ncbi.nlm.nih.gov/29455471/.

Rosen, H. (2012). Cracked tooth syndrome. The Journal of Prosthetic Dentistry, 47(1), 36-43. Recuperado de https://pubmed.ncbi.nlm.nih.gov/7033512

Sánchez, J. Jiménez, A., y Gale, G. (2018). Resistencia compresiva de dientes con conductos amplios restaurados con dos técnicas. Rev Clin Peridoncia Implantol Rehabil Oral, 11(1), 73-76. Recuperado de https://scielo.conicyt.cl/scielo.php?script=sci_arttext\&pid=S071901072018000100020\&Ing=es.

Sebeena, M., Boopathi, T., y Arjun, D. (2012). Diagnosis of cracked tooth. J Pharm Bioallied Sci, 4(2), 242-244. Recuperado de https://www.ncbi.nlm.gov/pmc/articles/PMC3467890/

Sin-Young, K., Su-Hyun, K., Soo-Bin, C.H., Gyung-Ok, L., y Sung-Eun, Y. (2013) Different Treatment Protocols for Different Pulpal and Periapical Diagnoses of 72 Cracked Teeth. J Endod, 39, 449-452. Recuperado de https://pubmed.ncbi.nlm.nih.gov/23522534/

Soares de Toubes, K.M., Moreira, L., y Cota, L. (2020). Optimization of Results for Cracked Teeth Using CAD-CAM System: A Case Series. Iranian Endodontic Journal, $\quad$ 1(15), 33-34. Recuperado de https://journals.sbmu.ac.ir/iej/article/view/26731

Sung-Eun, J., A-Ra, J., y Sin-Young, K. (2017). Analysis of the characteristics of cracked teeth and evaluation of pulp status according to periodontal probing depth. $B C M$ Oral Health, 17(1), $135 . \quad$ Recuperado de https://www.ncbi.nlm.nih.gov/pmc/articles/PMC5704503/

Weisburd, M.E., Giménez, J., Gutiérrez, E., Tomaghelli, E., y Perdomo, I. (2017). Síndrome del diente fisurado. Reporte de un caso. Repositorio Institucional de la UNLP. Recuperado de https://sedici.unlp.edu.ar/handle/10915/64145. 
West, N.X. (2008). Dentine hypersensitivity: preventive and therapeutic approaches to treatment. Periodont, 48(1), 31-41. Recuperado de https://pubmed.ncbi.nlm.nih.gov/18715354/

Yaping, Y., Gongpei, C., y Fang, H. (2019). Biting pain reproduced by the Tooth Slooth: an aid for early diagnosis of cracked tooth. Quintessence International, 1(50), 8287.

Recuperado de https://www.researchgate.net/publication/330309778_Biting_pain_reproduced_b y_the_Tooth_Slooth_an_aid_for_early_diagnosis_of_cracked_tooth

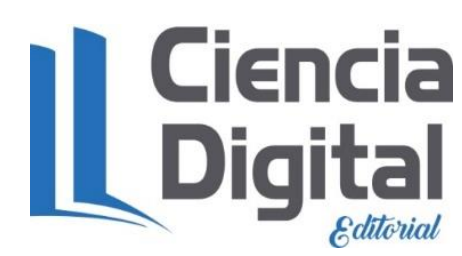




\section{PARA CITAR EL ARTÍCULO INDEXADO.}

Magariño Abreus, L. del R., Roque Batista, T., de León Ramírez, L. L., \& López González, E. (2021). Síndrome del Diente Fisurado: una actualización imprescindible . Anatomía Digital, 4(3), 87-101. https://doi.org/10.33262/anatomiadigital.v4i3.1758

\section{Liencia}

El artículo que se publica es de exclusiva responsabilidad de los autores y no necesariamente reflejan el pensamiento de la Revista Anatomía Digital.

El artículo queda en propiedad de la revista y, por tanto, su publicación parcial y/o total en otro medio tiene que ser autorizado por el director de la Revista Anatomía Digital.
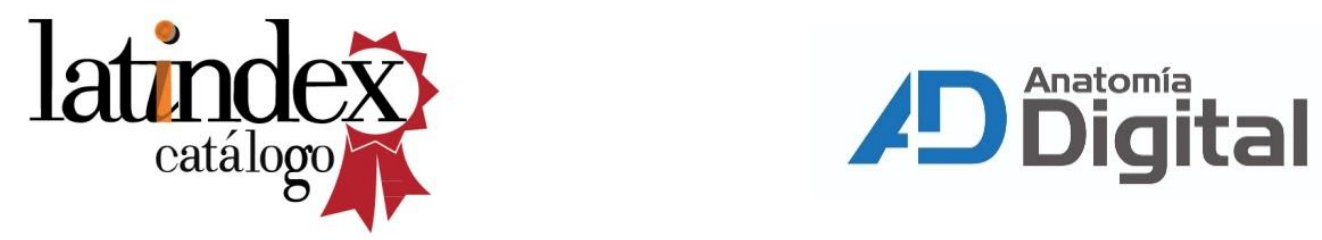